\title{
A Sulfoxide-Promoted, Catalytic Method for the Regioselective Synthesis of Allylic Acetates from Monosubstituted Olefins via C-H Oxidation.
}

\author{
Mark S. Chen and M. Christina White*
}

Department of Chemistry \& Chemical Biology, Harvard University, Cambridge, Massachusetts 02138.

General Information. Unless stated otherwise, reactions were conducted with stirring in $2 \mathrm{~mL}(0.2 \mathrm{mmol}$ reactions) or $40 \mathrm{~mL}$ (1.0 mmol reactions) VWRbrand borosilicate glass vials with red PTFE-faced silicone lined plastic screw caps or teflon-lined solid caps (respectively) under an air atmosphere. Vials were used as received (no cleansing or drying was done prior to reaction). All commercially obtained reagents (Sigma-Aldrich Chemical Company, unless stated otherwise) were used as received: anhydrous (Sure/Seal) DMSO; $4 \AA$ (powered, $<5$ micron) activated molecular sieves; 1,4-benzoquinone; glacial acetic acid (Mallinckrodt Chemicals); $\mathrm{Pd}(\mathrm{OAc})_{2}$ (Strem Chemicals); $\mathrm{Pd}\left(\mathrm{O}_{2} \mathrm{CCF}_{3}\right)_{2}$ (Strem Chemicals). Both palladium sources were stored in a glove box under a nitrogen atmosphere. $\mathrm{Pd}(\mathrm{OAc})_{2}$ was weighed in the air whereas $\mathrm{Pd}\left(\mathrm{O}_{2} \mathrm{CCF}_{3}\right)_{2}\left[\mathrm{Pd}(\mathrm{TFA})_{2}\right]$ was weighed in the glove box. Solvents tetrahydrofuran (THF), diethyl ether $\left(\mathrm{Et}_{2} \mathrm{O}\right)$, ethylene glycol dimethyl ether (DME), methylene chloride $\left(\mathrm{CH}_{2} \mathrm{Cl}_{2}\right)$, toluene, benzene were purified prior to use by passage through a bed of activated alumina (Glass Contour, Laguna Beach, California). Acetonitrile $\left(\mathrm{CH}_{3} \mathrm{CN}\right)$ was distilled from $\mathrm{CaH}_{2}$. Gas chromatographic (GC) analyses were performed on Agilent Technologies 6890N Series instrument equipped with FID detectors using a HP-5 (5\%Phenyl)-methylpolysiloxane column $(30 \mathrm{~m}, 0.32 \mathrm{~mm}, 0.25 \mu \mathrm{m})$. GC yields reported relative to an internal standard (nitrobenzene) and corrected for response factor variations. Unless otherwise noted, linear to branched allylic acetate ratios $[\mathrm{L}: \mathrm{B}]$ were determined by GC analysis of the crude and were not corrected for small response factor variations. Retention times for the branched isomers were determined by independent synthesis using catalyst $\mathbf{1}$ (see general procedure for catalyst 1, equations 2 and 3) which gave the branched as the major products for substrates constituting entries 3-9 in Table 1 or as mixtures for entry 1 and 10 ([L:B] $=4: 1,[\mathrm{~L}: \mathrm{B}]=2: 1$, respectively). Thinlayer chromatography (TLC) was conducted with E. Merck silica gel 60 F254 precoated plates $(0.25 \mathrm{~mm})$ and visualized with UV and potassium permanganate staining. Reaction progression was monitored by both GC and TLC analysis. Flash column chromatography was performed as described by Still et al. ${ }^{1}$ using EM reagent silica gel 60 (230-400 mesh). ${ }^{1} \mathrm{H}$ NMR spectra were recorded on a Varian Mercury-400 (400 MHz) spectrometer and are reported in ppm using solvent as an internal standard $\left(\mathrm{CDCl}_{3}\right.$ at $\left.7.26 \mathrm{ppm}\right)$. Data reported as: $\mathrm{s}=$ singlet, $\mathrm{d}=$ doublet, $\mathrm{t}=$ triplet, $\mathrm{q}=$ quartet, $\mathrm{m}=$ multiplet, $\mathrm{br}=$ broad, app = apparent; coupling constant(s) in $\mathrm{Hz}$; integration. Ratios of $E$ to $Z$ isomers were determined by ${ }^{1} \mathrm{H}$ NMR analysis of the crude upon workup and are based on integration of the allylic hydrogens of the acetoxy-bearing carbon of the two isomers. Assignments of all isomers were confirmed by gCOSY experiments. Proton-decoupled ${ }^{13} \mathrm{C}$ NMR spectra were recorded on a Varian Mercury$400(100 \mathrm{MHz})$ spectrometer and are reported in ppm using solvent as an internal standard $\left(\mathrm{CDCl}_{3}\right.$ at $\left.77.0 \mathrm{ppm}\right)$. IR spectra were recorded as thin films on $\mathrm{NaCl}$ plates on a Matterson FTIR 3000 and are reported in frequency of absorption $\left(\mathrm{cm}^{-1}\right)$. High resolution mass spectra were obtained at the Harvard University Mass Spectrometry Laboratory. Elemental analysis was conducted at Desert Analytics, Tucson, Arizona.

\section{Synthesis and Analytical Data for Catalyst 1.}

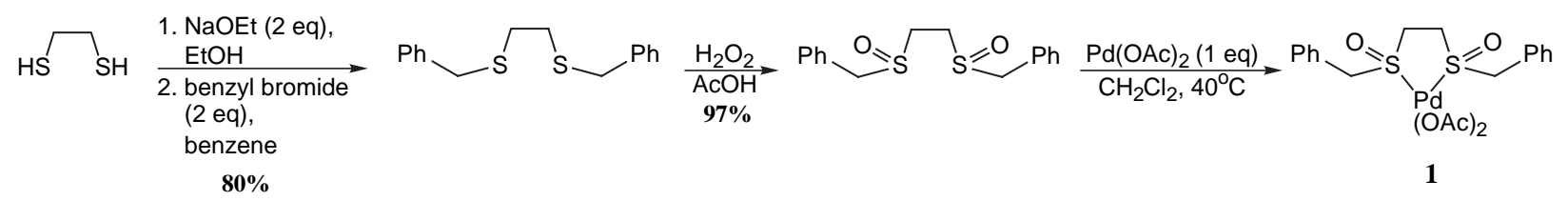

\section{1,2-bis(benzylthio)ethane ${ }^{2}$ :}

A flame-dried $500 \mathrm{~mL}$ round bottom flask was charged sequentially with a stir bar, NaOEt (2.94g, $43.2 \mathrm{mmol})$, and EtOH (105 mL) under a $\mathrm{N}_{2}$ atmosphere. 1,2-ethanedithiol (1.8 $\left.\mathrm{mL}, 21.4 \mathrm{mmol}\right)$ was added dropwise via syringe to the NaOEt solution and the mixture was allowed to stir for $20 \mathrm{~min}$. A solution of benzyl bromide $(5 \mathrm{~mL}, 42.0 \mathrm{mmol})$ in benzene $(63 \mathrm{~mL})$ was added dropwise via cannula. The reaction was allowed to stir overnight. Solvents were removed under house vacuum $\left(\sim 25\right.$ torr) with mild heating $\left(40^{\circ} \mathrm{C}\right)$. The residue was diluted with $\mathrm{CHCl}_{3}(400 \mathrm{~mL})$ and washed with brine $(150 \mathrm{~mL})$. The brine was extracted three times with $\mathrm{CHCl}_{3}(3 \times 150 \mathrm{~mL})$. The organic layers 
were combined, dried over $\mathrm{MgSO}_{4}$, filtered and concentrated in vacuo to give a yellow oil. The yellow oil was suspended in EtOH $(40 \mathrm{~mL})$ and allowed to sit at $0^{\circ} \mathrm{C}$ for $\sim 1 \mathrm{~h}$. A white solid crashed out and was washed with EtOH until the supernatant was clear $(3 \times 30 \mathrm{~mL})$. The solid was dried under high vacuum for $7 \mathrm{~h}$ to give 1,2bis(benzylthio)ethane in $80 \%$ yield $(4.7 \mathrm{~g}, 17.1 \mathrm{mmol}) .{ }^{1} \mathrm{H} \mathrm{NMR}\left(400 \mathrm{MHz}, \mathrm{CDCl}_{3}\right) \delta 7.28(\mathrm{~m}, 10 \mathrm{H}), 3.69(\mathrm{~s}, 4 \mathrm{H})$, $2.58(\mathrm{~s}, 4 \mathrm{H}) ;{ }^{13} \mathrm{C} \mathrm{NMR}\left(100 \mathrm{MHz}, \mathrm{CDCl}_{3}\right) \delta 138.1,128.8,128.5,127.0,36.2,30.9$. HRMS $\left(\mathrm{EI}^{+}\right) \mathrm{m} / z$ calc'd for $\left[\mathrm{C}_{16} \mathrm{H}_{18} \mathrm{~S}_{2}\right]^{+}: 274.0850$, found 274.0842 .

\section{1,2-bis(benzylsulfinyl)ethane ${ }^{3}$ :}

A $100 \mathrm{~mL}$ round bottom flask was charged with a stir bar, $2.5 \mathrm{~g}(9.1 \mathrm{mmol})$ of 1,2-bis(benzylthio)ethane, and 22 $\mathrm{mL}$ of glacial acetic acid. The mixture was allowed to stir at room temperature until it became homogeneous at which time it was cooled to $0^{\circ} \mathrm{C}$ and $\mathrm{H}_{2} \mathrm{O}_{2}(50 \mathrm{wt} \%$ solution, $1.05 \mathrm{~mL}, 18.22 \mathrm{mmol})$ was added dropwise. The reaction was allowed to come to room temperature and was stirred overnight. The acetic acid was removed with mild heating $\left(50^{\circ} \mathrm{C}\right)$ under high vacuum and the white solid was washed with $\mathrm{EtOH}$ until the washings were clear $(3$ x $40 \mathrm{~mL}$ ). The white solid was dried under high vacuum overnight to give $2.71 \mathrm{~g}(8.84 \mathrm{mmol})$ of 1,2 bis(benzylsulfinyl)ethane in $97 \%$ yield. ${ }^{1} \mathrm{H}$ NMR $\left(400 \mathrm{MHz}, \mathrm{CDCl}_{3}\right) \delta 7.36(\mathrm{~m}, 5 \mathrm{H}), 7.27(\mathrm{~m}, 5 \mathrm{H}), 4.03$ (app dq, $J$ $=3.6,13.2 \mathrm{~Hz}, 4 \mathrm{H}), 3.03(\mathrm{~m}, 2 \mathrm{H}), 2.87(\mathrm{~m}, 2 \mathrm{H}) ;{ }^{13} \mathrm{C} \mathrm{NMR}\left(100 \mathrm{MHz}, \mathrm{CDCl}_{3}\right) \delta 130.1,129.1,128.9,128.8,128.7$, 128.69, 58.9, 58.5, 43.5, 42.7; IR (film, $\left.\mathrm{cm}^{-1}\right): 2959,2913,1454,1024,768,698$; HRMS (EI) $\mathrm{m} / \mathrm{z}$ calc'd for $\left[\mathrm{C}_{16} \mathrm{H}_{18} \mathrm{O}_{2} \mathrm{~S}_{2}\right]^{+}:$306.0748, found 306.0751 .

\section{Catalyst $1^{4}$ :}

A flame dried $250 \mathrm{~mL}$ flask was charged with $1.5 \mathrm{~g}$ (4.89 mmol) of 1,2-bis(benzylsulfinyl)ethane, $70 \mathrm{~mL}$ of $\mathrm{CH}_{2} \mathrm{Cl}_{2}$, and $1.1 \mathrm{~g}(4.89 \mathrm{mmol})$ of $\mathrm{Pd}(\mathrm{OAc})_{2}$. The mixture was stirred at reflux under a nitrogen atmosphere for $22 \mathrm{~h}$. During this time the reaction was observed to turn from a heterogeneous orange brown mixture to a homogeneous, dark red solution. The solution was concentrated in vacuo and dried over a $\mathrm{N}_{2}$ stream for $12 \mathrm{~h}$ to give a dark red solid that was used without further purification. IR (film, cm-1): 3032, 1736, 1555, 1495, 1454, 1413, 1231, 1070, 1045, 1030, 766, 698. MS (ESI) $\mathrm{m} / \mathrm{z} 413\left(\left[\mathrm{M}-(\mathrm{OAc})_{2}+\mathrm{H}\right]^{+}\right.$, center of isotope cluster); Anal. Calcd for $\left[\mathrm{C}_{20} \mathrm{H}_{24} \mathrm{O}_{6} \mathrm{PdS}_{2}\right]$ (\%): C, 45.24; H, 4.56; Pd, 20.04; S, 12.08. Found (\%): C, 45.61; H, 4.53; Pd, 20.63; S, 12.81. 
Table 1. The effect of DMSO and bis-sulfoxide ligation on Pd(II) catalyzed oxidations of terminal olefins

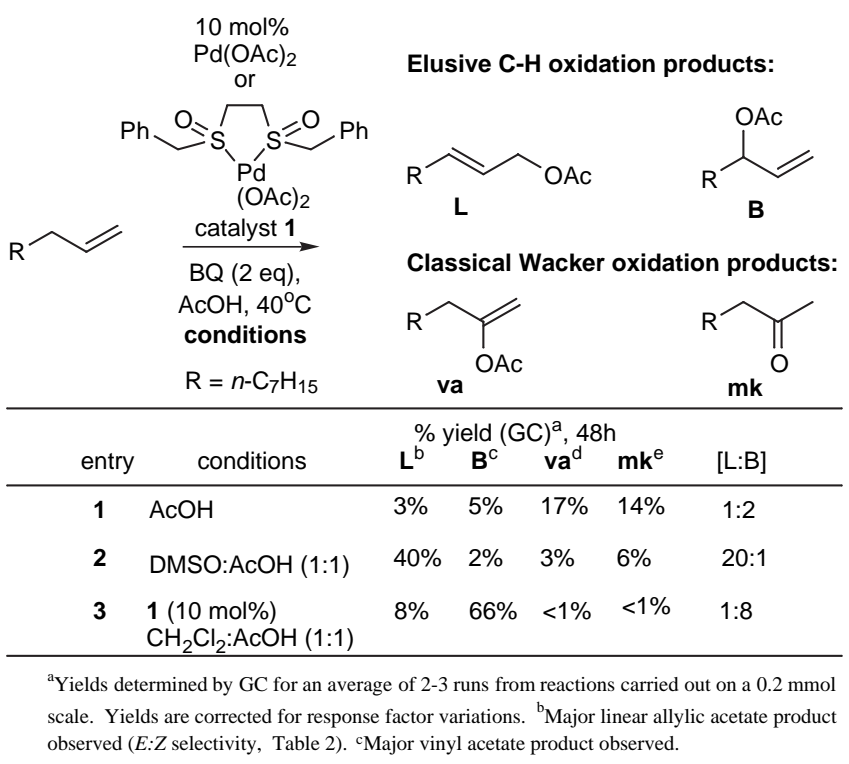

General procedure for Tables 1, S1-S8: Vials ( $2 \mathrm{~mL}$, borosilicate) were sequentially charged with the following solids: $\mathrm{Pd}(\mathrm{OAc})_{2}(4.5 \mathrm{mg}, 0.02 \mathrm{mmol}, 10 \mathrm{~mol} \%)$ or Pd(TFA) $)_{2}(6.6 \mathrm{mg}, 0.02 \mathrm{mmol}, 10 \mathrm{~mol} \%)$ or catalyst $1(10.6 \mathrm{mg}$, $0.02 \mathrm{mmol}, 10 \mathrm{~mol} \%)$, benzoquinone ( $43.2 \mathrm{mg}, 0.4 \mathrm{mmol}, 2 \mathrm{eq})$, and when indicated $4 \AA \mathrm{MS}$ (43 mg). Separate vials $(2 \mathrm{~mL}$, borosilicate) were sequentially charged with the following liquids: 1-decene ( $28 \mathrm{mg}, 38 \mu \mathrm{L}, 0.2 \mathrm{mmol}$, $1 \mathrm{eq})$, nitrobenzene $(9.9 \mathrm{mg}, 8 \mu \mathrm{L}, 0.08 \mathrm{mmol}, 40 \mathrm{~mol} \%), 1.2 \mathrm{~mL} \mathrm{AcOH}$ or $0.6 \mathrm{~mL} \mathrm{DMSO} / 0.6 \mathrm{~mL}$ AcOH or 0.6 $\mathrm{mL}$ solvent/ $0.6 \mathrm{~mL} \mathrm{AcOH}$. Aliquots were taken from the liquid vials $\left(\sim 10 \mu \mathrm{L}\right.$ filtered with $\mathrm{Et}_{2} \mathrm{O}$ through a short pipette plug of silica) to determine GC initial ratios of 1-decene to the nitrobenzene internal standard. The liquids were transferred via pipette into the appropriate solids vials, charged with a stir bar, capped and allowed to heat at $40^{\circ} \mathrm{C}$ with stirring. Aliquots were taken (as above) at $\mathrm{t}=24 \mathrm{~h}, \mathrm{t}=48 \mathrm{~h}$ (shown) and $\mathrm{t}=72 \mathrm{~h}$ to determine GC yields. Each product was independently synthesized via known methods (L: Horner-Wadsworth-Emmons olefination of octyl aldehyde followed by reduction and acetylation, B: vinyl magnesium bromide alkylation of octyl aldehyde followed by acetylation, va: 2-decanone, isopropenyl acetate, $p$-toluenesulfonic acid ${ }^{5}$, mk: Aldrich). Response factors relative to decene were determined for each product. Note: Table $S 4$ done on half the scale indicated above (i.e. 1-decene: $14.0 \mathrm{mg}, 19 \mu \mathrm{L}, 0.1 \mathrm{mmol}$, etc.).

Table S1. $4 \AA$ MS screen with $10 \mathrm{~mol} \% \mathrm{Pd}(\mathrm{OAc})_{2}$.

\begin{tabular}{|c|c|c|c|c|c|}
\hline \multirow[b]{2}{*}{ entry } & \multirow[b]{2}{*}{ conditions } & \multicolumn{4}{|c|}{$\%$ yield (GC), $48 \mathrm{~h}$} \\
\hline & & L & B & va & mk \\
\hline 1 & $\mathrm{AcOH}$ & $3 \%$ & $5 \%$ & $17 \%$ & $14 \%$ \\
\hline 2 & $\mathrm{AcOH} / 4 \AA \AA \mathrm{MS}$ & $15 \%$ & $5 \%$ & $11 \%$ & $21 \%$ \\
\hline 3 & DMSO:AcOH (1:1) & $40 \%$ & $2 \%$ & $3 \%$ & $6 \%$ \\
\hline 4 & $\begin{array}{l}\text { DMSO:AcOH }(1: 1) / \\
4 \AA ̊ M S\end{array}$ & $47 \%$ & $2 \%$ & $2 \%$ & $5 \%$ \\
\hline
\end{tabular}

Table S2. $4 \AA \AA$ MS screen with $10 \mathrm{~mol} \% \mathrm{Pd}(\mathrm{TFA})_{2}$

\begin{tabular}{|llllll|}
\hline & & \multicolumn{5}{c|}{$\%$ yield (GC), $48 \mathrm{~h}$} \\
entry & \multicolumn{1}{c}{ conditions } & L & B & va & mk \\
\hline $\mathbf{1}$ & AcOH & $3 \%$ & $4 \%$ & $11 \%$ & $29 \%$ \\
$\mathbf{2}$ & DMSO:AcOH (1:1) & $25 \%$ & $3 \%$ & $4 \%$ & $16 \%$ \\
$\mathbf{3}$ & DMSO:AcOH (1:1)/ & $46 \%$ & $2 \%$ & $2 \%$ & $6 \%$ \\
& $4 \AA \AA \mathrm{MS}$ & & & & \\
\hline
\end{tabular}


Table S3. Solvent screen (solvent:AcOH, 1:1) with $10 \mathrm{~mol} \%$ $\mathrm{Pd}(\mathrm{OAc})_{2}, \mathrm{BQ}(2 \mathrm{eq}), 40^{\circ} \mathrm{C}$.

\begin{tabular}{|c|c|c|c|c|c|}
\hline \multirow[b]{2}{*}{ entry } & \multirow[b]{2}{*}{ solvent } & \multicolumn{3}{|c|}{$\%$ yield $(\mathrm{GC}), 48 \mathrm{~h}$} & \multirow[b]{2}{*}{ mk } \\
\hline & & $\mathbf{L}$ & B & va & \\
\hline 1 & $\mathrm{AcOH}$ & $3 \%$ & $4 \%$ & $18 \%$ & $12 \%$ \\
\hline 2 & DMSO & $40 \%$ & $2 \%$ & $3 \%$ & $4 \%$ \\
\hline 3 & DMF & $3 \%$ & $<1 \%$ & $3 \%$ & $<1 \%$ \\
\hline 4 & dioxane & $<1 \%$ & $2 \%$ & $20 \%$ & $6 \%$ \\
\hline 5 & $\mathrm{CH}_{3} \mathrm{CN}$ & $8 \%$ & $5 \%$ & $2 \%$ & $2 \%$ \\
\hline 6 & THF & $<1 \%$ & $1 \%$ & $13 \%$ & $3 \%$ \\
\hline 7 & $\mathrm{Et}_{2} \mathrm{O}$ & $<1 \%$ & $1 \%$ & $14 \%$ & $5 \%$ \\
\hline 8 & DME & $<1 \%$ & $2 \%$ & $16 \%$ & $6 \%$ \\
\hline 9 & $\mathrm{CH}_{2} \mathrm{Cl}_{2}$ & $<1 \%$ & $2 \%$ & $10 \%$ & $3 \%$ \\
\hline 10 & Benzene & $<1 \%$ & $1 \%$ & $10 \%$ & $2 \%$ \\
\hline 11 & Toluene & $2 \%$ & $2 \%$ & $10 \%$ & $2 \%$ \\
\hline
\end{tabular}

Table S5. Oxidant screen with $10 \mathrm{~mol} \% \mathrm{Pd}(\mathrm{OAc})_{2}, 4 \AA \mathrm{MS}$, DMSO:AcOH (1:1), $40^{\circ} \mathrm{C}$.

\begin{tabular}{|c|c|c|c|c|c|}
\hline \multirow[b]{2}{*}{ entry } & \multirow[b]{2}{*}{ [0] } & \multicolumn{4}{|c|}{$\%$ yield $(\mathrm{GC}), 48 \mathrm{~h}$} \\
\hline & & $\mathbf{L}$ & B & va & mk \\
\hline 1 & $B Q(2 \mathrm{eq})$ & $47 \%$ & $2 \%$ & $2 \%$ & $4 \%$ \\
\hline 2 & $\mathrm{Cu}(\mathrm{OAc})_{2}(2 \mathrm{eq})$ & $2 \%$ & $1 \%$ & $<1 \%$ & $1 \%$ \\
\hline 3 & $\begin{array}{l}\mathrm{BQ}(20 \mathrm{~mol} \%) \\
\mathrm{MnO}_{2}(2 \mathrm{eq})\end{array}$ & $31 \%$ & $2 \%$ & $1 \%$ & $4 \%$ \\
\hline 4 & $\begin{array}{l}\mathrm{Cu}(\mathrm{OAc})_{2} \\
(5 \mathrm{~mol} \%) \\
\mathrm{O}_{2}\end{array}$ & $17 \%$ & $3 \%$ & $1 \%$ & $3 \%$ \\
\hline 5 & $\begin{array}{l}\mathrm{Cu}(\mathrm{OAc}) 2 \\
(5 \mathrm{~mol} \%) \\
\mathrm{BQ}(10 \mathrm{~mol} \%) \\
\mathrm{O}_{2}\end{array}$ & $32 \%$ & $3 \%$ & $1 \%$ & $4 \%$ \\
\hline 6 & $\mathrm{O}_{2}$ & $6 \%$ & $2 \%$ & $<1 \%$ & $1 \%$ \\
\hline
\end{tabular}

Table S4. Solvent screen (solvent:AcOH, 1:1) with $10 \mathrm{~mol} \%$ catalyst $1, B Q\left(2\right.$ eq), $40^{\circ} \mathrm{C}$.

\begin{tabular}{|c|c|c|c|c|c|}
\hline \multirow[b]{2}{*}{ entry } & \multirow[b]{2}{*}{ solvent } & \multicolumn{4}{|c|}{$\%$ yield (GC), $48 \mathrm{~h}$} \\
\hline & & $\mathbf{L}$ & B & va & mk \\
\hline 1 & $\mathrm{AcOH}$ & $17 \%$ & $59 \%$ & $<1 \%$ & $<1 \%$ \\
\hline 2 & DMSO & $12 \%$ & $7 \%$ & $<1 \%$ & $<1 \%$ \\
\hline 3 & DMF & $13 \%$ & $50 \%$ & $<1 \%$ & $<1 \%$ \\
\hline 4 & $\mathrm{CH}_{3} \mathrm{CN}$ & $6 \%$ & $3 \%$ & $2 \%$ & $<1 \%$ \\
\hline 5 & dioxane & $9 \%$ & $61 \%$ & $<1 \%$ & $<1 \%$ \\
\hline 6 & THF & $7 \%$ & $53 \%$ & $<1 \%$ & $<1 \%$ \\
\hline 7 & $\mathrm{Et}_{2} \mathrm{O}$ & $8 \%$ & $54 \%$ & $<1 \%$ & $<1 \%$ \\
\hline 8 & DME & $8 \%$ & $57 \%$ & $<1 \%$ & $<1 \%$ \\
\hline 9 & $\mathrm{CH}_{2} \mathrm{Cl}_{2}$ & $9 \%$ & $68 \%$ & $<1 \%$ & $<1 \%$ \\
\hline 10 & Benzene & $8 \%$ & $63 \%$ & $<1 \%$ & $<1 \%$ \\
\hline 11 & Toluene & $9 \%$ & $59 \%$ & $<1 \%$ & $<1 \%$ \\
\hline
\end{tabular}

Table S6. Benzoquinone equivalents screen with $10 \mathrm{~mol} \%$ $\mathrm{Pd}(\mathrm{OAc})_{2}, 4 \AA \mathrm{MS}$, DMSO:AcOH $(1: 1), 40^{\circ} \mathrm{C}$.

\begin{tabular}{|c|c|c|c|c|c|}
\hline \multirow[b]{2}{*}{ entry } & \multirow[b]{2}{*}{$B Q(e q)$} & \multicolumn{4}{|c|}{$\%$ yield (GC), $48 \mathrm{~h}$} \\
\hline & & $\mathbf{L}$ & B & va & mk \\
\hline 1 & 1.0 & $36 \%$ & $4 \%$ & $1 \%$ & $4 \%$ \\
\hline 2 & 1.5 & $43 \%$ & $2 \%$ & $2 \%$ & $4 \%$ \\
\hline 3 & 2.0 & $46 \%$ & $2 \%$ & $2 \%$ & $4 \%$ \\
\hline 4 & 2.5 & $44 \%$ & $2 \%$ & $2 \%$ & $4 \%$ \\
\hline 5 & 3.0 & $43 \%$ & $2 \%$ & $2 \%$ & $4 \%$ \\
\hline
\end{tabular}


Table S7. DMSO:AcOH ratio with $10 \mathrm{~mol} \% \mathrm{Pd}(\mathrm{OAc})_{2}$, $4 \AA \mathrm{AS}, \mathrm{BQ}(2 \mathrm{eq}), 40^{\circ} \mathrm{C}$.

\begin{tabular}{|c|c|c|c|c|c|}
\hline \multirow[b]{2}{*}{ entry } & \multirow[b]{2}{*}{ DMSO: AcOH } & \multicolumn{4}{|c|}{$\%$ yield (GC), $48 \mathrm{~h}$} \\
\hline & & $\mathbf{L}$ & B & va & mk \\
\hline 1 & $5: 1$ & $5 \%$ & $<1 \%$ & $<1 \%$ & --- \\
\hline 2 & $2: 1$ & $24 \%$ & $1 \%$ & $1 \%$ & $2 \%$ \\
\hline 3 & $1: 1$ & $44 \%$ & $2 \%$ & $1 \%$ & $4 \%$ \\
\hline 4 & $1: 2$ & $55 \%$ & $5 \%$ & $2 \%$ & $6 \%$ \\
\hline 5 & $1: 5$ & $50 \%$ & $7 \%$ & $4 \%$ & $7 \%$ \\
\hline
\end{tabular}

Table S8. Reaction temperature screen with $10 \mathrm{~mol} \%$ $\mathrm{Pd}(\mathrm{OAc})_{2}, \mathrm{BQ}(2 \mathrm{eq}), 4 \AA \mathrm{AS}$, and DMSO:AcOH (1:1).

\begin{tabular}{|c|c|c|c|c|c|}
\hline \multirow[b]{2}{*}{ entry } & \multirow[b]{2}{*}{ temperature } & \multicolumn{4}{|c|}{$\%$ yield (GC), $48 \mathrm{~h}$} \\
\hline & & $\mathbf{L}$ & B & va & mk \\
\hline 1 & $25^{\circ} \mathrm{C}$ & $13 \%$ & $<1 \%$ & $<1 \%$ & $1 \%$ \\
\hline 2 & $40^{\circ} \mathrm{C}$ & $45 \%$ & $2 \%$ & $2 \%$ & $4 \%$ \\
\hline 3 & $50^{\circ} \mathrm{C}$ & $27 \%$ & $9 \%$ & $2 \%$ & $4 \%$ \\
\hline 4 & $60^{\circ} \mathrm{C}$ & $21 \%$ & $9 \%$ & $2 \%$ & $4 \%$ \\
\hline
\end{tabular}


Table 2. Allylic oxidation of terminal olefins to $(E)$-allylic acetates. ${ }^{a}$

(1)

${ }^{a}$ All data reported ([L:B], [E:Z] ratios, yields) based on an average of two runs. ${ }^{b} 10 \mathrm{~mol} \% \operatorname{Pd}(\mathrm{TFA})_{2}$.

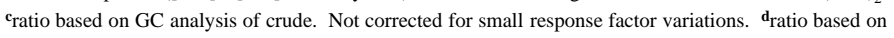
${ }^{1} \mathrm{H}$ NMR analysis of the crude. ${ }^{\mathrm{e}}$ Isolated yields after chromatography, from reactions carried out on a 1.0 mmol scale $(0.17 \mathrm{M})$. Unpurified commercial DMSO and glacial acetic acid were used, without inert atmosphere techniques. $[\mathrm{L}: \mathrm{B}]$ and $[E: Z]$ ratios obtained after chromatography were generally higher. See Supporting Information for details. 
Terminal olefin substrates: The following terminal olefin substrates used were obtained from commercial sources: 1-decene (entry 3); allylbenzene (entry 10); ethyl 4-pentenoate (entry 2, Lancaster Synthesis). The remainder of the substrates were prepared via previously reported literature routes: 2-allylcyclohexanone ethylene ketal (entry 1$)^{6}, 5$ (tert-butyldiphenylsiloxy)-1-pentene (entry 4) ${ }^{7}$, 1-benzyloxy-4-pentene (entry 5) ${ }^{8}$, 2-hydroxy-benzoic acid hex-5enyl ester (entry 6/7) ${ }^{9}$, phenyl-carbamic acid hex-5-enyl ester (entry 8$)^{9}, N, N$-diethyl-6-heptenamide (entry 9$)^{10}$.

General procedure for Table 2: A $40 \mathrm{~mL}$ borosilicate glass vial was charged with the following solids: $\mathrm{Pd}(\mathrm{OAc})_{2}$ (22.4 mg, $0.1 \mathrm{mmol}, 10 \mathrm{~mol} \%)$ or Pd(TFA) $2(33.2 \mathrm{mg}, 0.1 \mathrm{mmol}, 10 \mathrm{~mol} \%)$, benzoquinone $(217 \mathrm{mg}, 2.0 \mathrm{mmol}, 2$ eq), and $4 \AA \mathrm{MS}(217 \mathrm{mg})$. In cases (entries $2, \mathbf{4}, \mathbf{5})$ where $\mathrm{Pd}(\mathrm{TFA})_{2}$ was used, $\mathrm{Pd}(\mathrm{OAc})_{2}$ gave slightly lower yields and/or [L:B] selectivities. To the solids vial was sequentially added the following: DMSO (3 mL), olefin substrate $(1.0 \mathrm{mmol}, 1 \mathrm{eq})$, AcOH $(3 \mathrm{~mL})$. The vial was charged with a stir bar, capped and allowed to heat at $40^{\circ} \mathrm{C}$. Aliquots were taken $\left(\sim 10 \mu \mathrm{L}\right.$ filtered through a silica plug with $\left.\mathrm{Et}_{2} \mathrm{O}\right)$ at $\mathrm{t}=24 \mathrm{~h}, 48 \mathrm{~h}$, and $72 \mathrm{~h}$ to determine linear:branched ratios [L:B]. For the majority of substrates examined (entries 3-9), the general trend observed was that of a slight decrease in $[\mathrm{L}: \mathrm{B}]$ ratios over the course of the reaction (see below). The reaction was quenched with saturated $\mathrm{NH}_{4} \mathrm{Cl}(10 \mathrm{~mL})$ and extracted with $\mathrm{CH}_{2} \mathrm{Cl}_{2}(3 \mathrm{x} 100 \mathrm{~mL})$. The combined organic layers were washed with $\mathrm{H}_{2} \mathrm{O}(2 \times 100 \mathrm{~mL})$, dried over $\mathrm{MgSO}_{4}$, filtered and concentrated in vacuo to give a brown oil. A crude ${ }^{1} \mathrm{H} \mathrm{NMR}$ was taken to determine $[E: Z]$ ratios. Purification of the crude by flash chromatography afforded the linear $E$-allylic acetates as the major products with trace amounts of the linear $Z$-allylic acetates and the branched acetates. All data reported in Table 1 is based on an average of 2 runs.

Entry 1: 72h. run 1,2: $[\mathrm{L}: \mathrm{B}]=>99: 1,[E: Z]=>20: 1$ (branched or $Z$ isomers were not observed by $\mathrm{GC}$ or ${ }^{1} \mathrm{H}$ NMR). Purification by flash chromatography (10\% EtOAc/hexanes eluent) provided pure linear $(E)$-allylic acetate ( run 1 : $119 \mathrm{mg}, 0.49 \mathrm{mmol}, 49 \%$ yield; run 2: $120.5 \mathrm{mg}, 0.5 \mathrm{mmol}, 50 \%$ yield; average yield: $50 \%$ ).

${ }^{1} \mathrm{H}$ NMR $\left(400 \mathrm{MHz}, \mathrm{CDCl}_{3}\right) \delta 5.80(\mathrm{dd}, J=15.6,7.6 \mathrm{~Hz}, 1 \mathrm{H}), 5.61(\mathrm{dt}, J=15.6,6.0 \mathrm{~Hz}, 1 \mathrm{H}), 4.52(\mathrm{~d}$, $J=6.8 \mathrm{~Hz}, 2 \mathrm{H}), 3.90(\mathrm{~m}, 4 \mathrm{H}), 2.31(\mathrm{~m}, 1 \mathrm{H}), 2.05(\mathrm{~s}, 3 \mathrm{H}), 1.78-1.2(\mathrm{~m}, 8 \mathrm{H}) ;{ }^{13} \mathrm{C} \mathrm{NMR}\left(100 \mathrm{MHz}, \mathrm{CDCl}_{3}\right) \delta$ 170.9, 135.2, 125.1, 109.9, 65.3, 65.1, 64.9, 48.2, 35.2, 30.0, 24.3, 23.8, 21.0; IR (film, cm $\left.{ }^{-1}\right): 2936,2884$, 2864, 1740, 1447, 1233; HRMS (EI) $\mathrm{m} / z$ calc'd for $\left[\mathrm{C}_{13} \mathrm{H}_{20} \mathrm{O}_{4}\right]^{+}: 240.1362$, found 240.1369 .

Entry 2: 48 h. run $1,2:[\mathrm{L}: \mathrm{B}]=>20 ; 1,[E: Z]=13: 1$. Both ratios determined by ${ }^{1} \mathrm{H}$ NMR. Purification by flash chromatography (15\% EtOAc/hexanes eluent) provided the desired linear $(E)$-allylic acetate as the major product (run 1: $102.4 \mathrm{mg}, 0.55 \mathrm{mmol}, 55 \%$; run 2: $96.8 \mathrm{mg}, 0.52 \mathrm{mmol}, 52 \%$; average yield: $54 \%$ ). After column: [L:B] = $>20: 1 ;[E: Z]=16: 1$.

${ }^{1} \mathrm{H}$ NMR $\left(400 \mathrm{MHz}, \mathrm{CDCl}_{3}\right) \delta 5.88(\mathrm{dt}, J=15.6,6.8 \mathrm{~Hz}, 1 \mathrm{H}), 5.70(\mathrm{dt}, J=15.6,6.4 \mathrm{~Hz}, 1 \mathrm{H}), 4.55$ $(\mathrm{dd}, J=6.4,1.2 \mathrm{~Hz}, 2 \mathrm{H}), 4.14(\mathrm{q}, J=7.2 \mathrm{~Hz}, 3 \mathrm{H}), 3.09(\mathrm{dd}, J=6.8,0.8 \mathrm{~Hz}, 2 \mathrm{H}), 2.06(\mathrm{~s}, 3 \mathrm{H}), 1.26(\mathrm{t}, J=$ $7.2 \mathrm{~Hz}, 3 \mathrm{H}) ;{ }^{13} \mathrm{C} \mathrm{NMR}\left(100 \mathrm{MHz}, \mathrm{CDCl}_{3}\right) \delta 171.2,170.7,127.8,127.1,64.4,60.8,37.6,20.9,14.1$; IR (film, $\mathrm{cm}^{-1}$ ): 2984, 2943, 2911, 1740, 1447, 1383, 1367, 1233, 1175; HRMS (EI) $\mathrm{m} / z$ calc'd for $\left[\mathrm{C}_{9} \mathrm{H}_{14} \mathrm{O}_{4}\right]^{+}$: 186.0892, found 186.0898 .

Entry 3: 48h. run 1: $[\mathrm{L}: \mathrm{B}]=24: 1,[\mathrm{E}: \mathrm{Z}]=12: 1$; run 2: $[\mathrm{L}: \mathrm{B}]=24: 1,[\mathrm{E}: \mathrm{Z}]=11: 1$; average: $[\mathrm{L}: \mathrm{B}]=24: 1,[\mathrm{E}: \mathrm{Z}]=$ 12:1 (24 h, $[\mathrm{L}: \mathrm{B}]=40: 1)$. Purification by flash chromatography (3\% EtOAc/hexanes eluent) provided the desired linear $(E)$-allylic acetate as the major product (run $1: 108 \mathrm{mg}, 0.54 \mathrm{mmol} ; 54 \%$; run $2: 101 \mathrm{mg}, 0.51 \mathrm{mmol}, 51 \%$; average yield: $52 \%)$. After column: $[\mathrm{L}: \mathrm{B}]=22: 1,[E: Z]=13: 1$.

${ }^{1} \mathrm{H}$ NMR $\left(400 \mathrm{MHz}, \mathrm{CDCl}_{3}\right) \delta 5.77(\mathrm{dt}, J=15.2,6.8 \mathrm{~Hz}, 1 \mathrm{H}), 5.56(\mathrm{dt}, J=15.6,6.8 \mathrm{~Hz}, 1 \mathrm{H}), 4.50(\mathrm{~d}$, $J=6.4 \mathrm{~Hz}, 2 \mathrm{H}), 2.06(\mathrm{~s}, 3 \mathrm{H}), 1.4-1.2(\mathrm{~m}, 12 \mathrm{H}), 0.87(\mathrm{t}, J=6.8 \mathrm{~Hz}, 3 \mathrm{H}) ;{ }^{13} \mathrm{C} \mathrm{NMR}\left(100 \mathrm{MHz}, \mathrm{CDCl}_{3}\right) \delta$ 170.9, 136.8, 123.6, 65.3, 32.2, 31.8, 29.1, 28.8, 22.6, 21.0, 14.1; IR (film, $\mathrm{cm}^{-1}$ ): 3021, 2957, 2928, 2857, $1744,1460,1231$. HRMS (EI) $\mathrm{m} / z$ calc'd for $\left[\mathrm{C}_{12} \mathrm{H}_{22} \mathrm{O}_{2}\right]^{+}: 198.1620$, found 198.1623.

Entry 4: 72h. run 1: $[\mathrm{L}: \mathrm{B}]=33: 1,[E: Z]=10: 1$; run 2: $[\mathrm{L}: \mathrm{B}]=28: 1,[E: Z]=11: 1$; average: $[\mathrm{L}: \mathrm{B}]=31: 1,[E: Z]=$ $11: 1(24 \mathrm{~h},[\mathrm{~L}: \mathrm{B}]=71: 1,48 \mathrm{~h},[\mathrm{~L}: \mathrm{B}]=43: 1)$. Purification by flash chromatography (3\% EtOAc/hexanes eluent) provided the desired linear $(E)$-allylic acetate as the major product (run 1: $191.2 \mathrm{mg}, 0.50 \mathrm{mmol}, 50 \%$; run 2: 191.4, $0.50 \mathrm{mmol}, 50 \%$; average yield: 50\%). After column: $[\mathrm{L}: \mathrm{B}]=87: 1,[E: Z]=12: 1$. 
${ }^{1} \mathrm{H}$ NMR $\left(400 \mathrm{MHz}, \mathrm{CDCl}_{3}\right) \delta 7.66(\mathrm{~m}, 4 \mathrm{H}), 7.40(\mathrm{~m}, 6 \mathrm{H}), 5.77(\mathrm{dt}, J=14,6.8 \mathrm{~Hz}, 1 \mathrm{H}), 5.61(\mathrm{dt}, J=$ 14, $6.4 \mathrm{~Hz}, 1 \mathrm{H}), 4.5(\mathrm{~d}, J=6.4 \mathrm{~Hz}, 2 \mathrm{H}), 3.7(\mathrm{t}, J=6.4 \mathrm{~Hz}, 2 \mathrm{H}), 2.31$ (brq, J = 5.6 Hz, 2H), $2.05(\mathrm{~s}, 3 \mathrm{H})$, $1.04(\mathrm{~s}, 9 \mathrm{H}) ;{ }^{13} \mathrm{C} \mathrm{NMR}\left(100 \mathrm{MHz}, \mathrm{CDCl}_{3}\right) \delta 170.8,135.6,133.8,132.8,129.6,127.6,125.8,65.1,63.1,35.6$, 26.8, 21.0, 19.2; IR (film, $\mathrm{cm}^{-1}$ ): 3071, 3050, 2999, 2957, 2932, 2893, 2859, 1742, 1472, 1427, 1111, 1231; HRMS (CI, $\mathrm{NH}_{3}$ ) $m / z$ calc'd for $\mathrm{C}_{23} \mathrm{H}_{34} \mathrm{NO}_{3} \mathrm{Si}\left[\mathrm{M}+\mathrm{NH}_{4}\right]^{+}$: 400.2308, found 400.2306.

Entry 5: 48h. run 1: $[\mathrm{L}: \mathrm{B}]=31: 1,[E: Z]=11: 1$, run 2: $[\mathrm{L}: \mathrm{B}]=31: 1,[E: Z]=10: 1$; average: [L:B] = 31:1, [E:Z] $=11: 1(24 \mathrm{~h},[\mathrm{~L}: \mathrm{B}]=52: 1)$. Purification by flash chromatography $(5 \%$ EtOAc/hexanes eluent $)$ provided the desired linear $(E)$-allylic acetate as the major product (run 1: $133.0 \mathrm{mg}, 0.57 \mathrm{mmol}, 57 \%$; run 2: $133.2 \mathrm{mg}, 0.57 \mathrm{mmol}$, 57\%; average yield: $57 \%)$. After column: $[\mathrm{L}: \mathrm{B}]=41: 1 ;[E: Z]=12: 1$.

${ }^{1} \mathrm{H}$ NMR $\left(400 \mathrm{MHz}, \mathrm{CDCl}_{3}\right) \delta$ 7.37-7.26 (m, 5H), $5.8(\mathrm{dt}, J=15.2,6.8 \mathrm{~Hz}, 1 \mathrm{H}), 5.66(\mathrm{dt}, J=15.6,6.4$ $\mathrm{Hz}, 1 \mathrm{H}), 4.52(\mathrm{~d}, J=4.8 \mathrm{~Hz}, 2 \mathrm{H}), 4.51(\mathrm{~s}, 2 \mathrm{H}), 3.52(\mathrm{t}, J=6.4 \mathrm{~Hz}, 2 \mathrm{H}), 2.39(\mathrm{brq}, \mathrm{J}=6.4 \mathrm{~Hz}, 2 \mathrm{H}), 2.06(\mathrm{~s}$, $3 \mathrm{H}) ;{ }^{13} \mathrm{C}$ NMR $\left(100 \mathrm{MHz}, \mathrm{CDCl}_{3}\right) \delta 170.8,138.3,132.5,128.4,127.64,127.58,125.8,72.9,69.3,65.0$, 32.7, 21.0; IR (film, $\mathrm{cm}^{-1}$ ): 3088, 3063, 3030, 2936, 2859, 2793, 1740, 1454, 1364, 1233; HRMS (CI, NH ${ }_{3}$ $m / z$ calc'd for $\mathrm{C}_{14} \mathrm{H}_{22} \mathrm{NO}_{3}\left[\mathrm{M}+\mathrm{NH}_{4}\right]^{+}:$252.1600, found 252.1593.

Entry 6: 72 h. run 1,2: [L:B] = 14:1, $[E: Z]=11: 1(24 h,[L: B]=39: 1,48 h,[L: B]=20: 1)$. Purification by flash chromatography (5\% EtOAc/hexanes eluent) provided the desired linear $(E)$-allylic acetate as the major product (run 1: $172.0 \mathrm{mg}$, $0.62 \mathrm{mmol}, 62 \%$; run $2: 166.0 \mathrm{mg}, 0.60 \mathrm{mmol}, 60 \%$; average yield: $61 \%)$. After column: [L:B] = $14: 1,[E: Z]=12: 1$.

${ }^{1} \mathrm{H}$ NMR $\left(400 \mathrm{MHz}, \mathrm{CDCl}_{3}\right) \delta 10.8(\mathrm{~s}, 1 \mathrm{H}), 7.83(\mathrm{dd}, J=8.4,1.6 \mathrm{~Hz}, 1 \mathrm{H}), 7.46(\mathrm{ddd}, J=8.6,7.2,2.0$ $\mathrm{Hz}, 1 \mathrm{H}), 6.98(\mathrm{dd}, J=8.4,0.8 \mathrm{~Hz}, 1 \mathrm{H}), 6.88(\mathrm{ddd}, J=8.2,8.0,1.2 \mathrm{~Hz}, 1 \mathrm{H}), 5.8(\mathrm{dt}, J=15.2,6.8 \mathrm{~Hz}, 1 \mathrm{H})$, $5.64(\mathrm{dt}, J=15.2,6.4 \mathrm{~Hz}, 1 \mathrm{H}), 4.52(\mathrm{dd}, \mathrm{J}=6.2,0.8 \mathrm{~Hz}, 2 \mathrm{H}), 4.36(\mathrm{t}, J=6.4 \mathrm{~Hz}, 2 \mathrm{H}), 2.24(\mathrm{bq}, J=7.6 \mathrm{~Hz}$, 2H), $2.06(\mathrm{~s}, 3 \mathrm{H}), 1.90(\mathrm{~m}, 2 \mathrm{H}) ;{ }^{13} \mathrm{C} \mathrm{NMR}\left(100 \mathrm{MHz}, \mathrm{CDCl}_{3}\right) \delta 170.8,170.1,161.7,135.7,134.4,129.8$, $125.1,124.9,119.1,117.6,112.5,64.9,64.6,28.6,27.7,21.0 ;$ IR (film, $\left.\mathrm{cm}^{-1}\right): 3187,3156,2953,1740$, 1674, 1615, 1485, 1302, 1250. HRMS (EI) $m / z$ calc'd for $\left[\mathrm{C}_{15} \mathrm{H}_{18} \mathrm{O}_{5}\right]+:$ 278.1154, found 278.1152.

Entry 7: 72h. run 1: $[\mathrm{L}: \mathrm{B}]=16: 1,[E: Z]=12: 1$; run $2:[\mathrm{L}: \mathrm{B}]=17: 1,[E: Z]=13: 1$; average: [L:B] $=17: 1$, $[E: Z]=13: 1(24 \mathrm{~h},[\mathrm{~L}: \mathrm{B}]=40: 1,48 \mathrm{~h},[\mathrm{~L}: \mathrm{B}]=22: 1)$. Purification by flash chromatography $(15 \%$ EtOAc/hexanes eluent) provided the desired linear $(E)$-allylic acetate as the major product (run 1: $160.2 \mathrm{mg}, 0.55 \mathrm{mmol}, 55 \%$; run 2: $162.9 \mathrm{mg}, 0.56 \mathrm{mmol}, 56 \%$; average yield: $56 \%)$. After column: [L:B] = 18:1, $[E: Z]=13: 1$.

${ }^{1} \mathrm{H}$ NMR $\left(400 \mathrm{MHz}, \mathrm{CDCl}_{3}\right) \delta 7.78(\mathrm{dd}, J=8.0,2.0 \mathrm{~Hz}, 1 \mathrm{H}), 7.46(\mathrm{ddd}, J=8.4,7.6,1.6 \mathrm{~Hz}, 1 \mathrm{H})$, $6.97(\mathrm{~m}, 2 \mathrm{H}), 5.80(\mathrm{dt}, J=15.6,6.8 \mathrm{~Hz}, 1 \mathrm{H}), 5.63(\mathrm{dt}, J=15.2,6.4 \mathrm{~Hz}, 1 \mathrm{H}), 4.51(\mathrm{dd}, J=6.4,0.8 \mathrm{~Hz}, 2 \mathrm{H})$, $4.30(\mathrm{t}, J=6.4 \mathrm{~Hz}, 2 \mathrm{H}), 3.9(\mathrm{~s}, 3 \mathrm{H}), 2.23(\mathrm{q}, \mathrm{J}=8.0 \mathrm{~Hz}, 2 \mathrm{H}), 2.05(\mathrm{~s}, 3 \mathrm{H}), 1.85(\mathrm{~m}, 2 \mathrm{H}) ;{ }^{13} \mathrm{C} \mathrm{NMR}(400$ $\left.\mathrm{MHz}, \mathrm{CDCl}_{3}\right) \delta 170.8,166.2,159.1,134.9,133.4,131.5,124.7,120.2,120.1,112.0,65.0,64.0,55.9,28.7$, 27.9, 21.0; IR (film, $\mathrm{cm}^{-1}$ ): 3079, 3003, 2949, 2841, 1736 (br), 1601, 1493, 1302, 1250; HRMS (EI) m/z calc'd for $\left[\mathrm{C}_{16} \mathrm{H}_{20} \mathrm{O}_{5}\right]+:$ 292.1311, found 292.1318 .

Entry 8: 72h. run 1: $[\mathrm{L}: \mathrm{B}]=13: 1,[E: Z]=12: 1$; run $2:[\mathrm{L}: \mathrm{B}]=12: 1,[E: Z]=12: 1$; average: [L:B] $=13: 1$, $[E: Z]=12: 1(24 \mathrm{~h},[\mathrm{~L}: \mathrm{B}]=37: 1,48 \mathrm{~h},[\mathrm{~L}: \mathrm{B}]=21: 1)$. Purification by flash chromatography $(15 \%$ EtOAc/hexanes eluent) provided the desired linear $(E)$-allylic acetate as the major product (run 1: $183.0 \mathrm{mg}, 0.66 \mathrm{mmol}, 66 \%$; run 2: $170.2 \mathrm{mg}, 0.61 \mathrm{mmol}, 61 \%$; average yield: 64\%). After column: [L:B] = 14:1; [E:Z] = 12:1.

${ }^{1} \mathrm{H}$ NMR $\left(400 \mathrm{MHz}, \mathrm{CDCl}_{3}\right) \delta 7.38(\mathrm{bd}, J=7.6 \mathrm{~Hz}, 2 \mathrm{H}), 7.30(\mathrm{t}, J=7.2 \mathrm{~Hz}, 2 \mathrm{H}), 7.06(\mathrm{t}, J=7.2 \mathrm{~Hz}$, $1 \mathrm{H}), 6.69(\mathrm{brs}, 1 \mathrm{H}), 5.78(\mathrm{dt}, J=15.2,6.4 \mathrm{~Hz}, 1 \mathrm{H}), 5.6(\mathrm{dt}, J=15.6,7.2 \mathrm{~Hz}, 1 \mathrm{H}), 4.52(\mathrm{dd}, J=6.0,0.4 \mathrm{~Hz})$, $\left.4.17(\mathrm{t}, J=6.4 \mathrm{~Hz}, 2 \mathrm{H}), 2.18(\mathrm{brq}, J=7.2 \mathrm{~Hz}, 2 \mathrm{H}), 2.06(\mathrm{~s}, 3 \mathrm{H}), 1.78(\mathrm{~m}, 2 \mathrm{H}) ;{ }^{13} \mathrm{C} \mathrm{NMR}(100 \mathrm{MHz} \mathrm{CDCl})\right)$ $\delta 170.9,153.5,137.9,134.9,129.0,124.7,123.4,118.6,65.0,64.6,28.7,28.1,21.0 ;$ IR $\left(\right.$ film, $\left.\mathrm{cm}^{-1}\right): 3329$ (br), 3138, 3080, 3044, 2953, 2851, 1736 (br, shoulder 1715), 1601, 1539, 1445, 1225; HRMS (EI) m/z calc'd for $\left[\mathrm{C}_{15} \mathrm{H}_{19} \mathrm{NO}_{4}\right]^{+}:$277.1314, found 277.1315 .

Entry 9: 48h. run 1: $[\mathrm{L}: \mathrm{B}]=24: 1,[E: Z]=12: 1$; run $2:[\mathrm{L}: \mathrm{B}]=21: 1,[E: Z]=12: 1$; average: [L:B] $=23: 1$, $[E: Z]=12: 1(24 \mathrm{~h},[\mathrm{~L}: \mathrm{B}]=44: 1)$. Purification by flash chromatography $(35 \%$ EtOAc/hexanes eluent $)$ provided the desired linear $(E)$-allylic acetate as the major product (run 1: $148.0 \mathrm{mg}, 0.61 \mathrm{mmol}, 61 \%$; run $2: 150.5 \mathrm{mg}, 0.62$ mmol, $62 \%$ yield; average yield: $62 \%)$. After column: $[\mathrm{L}: \mathrm{B}]=21: 1,[E: Z]=12: 1$.

${ }^{1} \mathrm{H}$ NMR $\left(400 \mathrm{MHz}, \mathrm{CDCl}_{3}\right) \delta 5.75(\mathrm{dt}, J=16,6.4 \mathrm{~Hz}, 1 \mathrm{H}), 5.58(\mathrm{dt}, J=15.2,6.0 \mathrm{~Hz}, 1 \mathrm{H}), 4.5(\mathrm{~d}, J$ $=6.4 \mathrm{~Hz}, 2 \mathrm{H}), 3.36(\mathrm{q}, J=7.6 \mathrm{~Hz}, 2 \mathrm{H}), 3.28(\mathrm{q}, J=7.6 \mathrm{~Hz}, 2 \mathrm{H}), 2.28(\mathrm{t}, J=7.6 \mathrm{~Hz}, 2 \mathrm{H}), 2.11(\mathrm{brq}, J=7.2$ 
$\mathrm{Hz}, 2 \mathrm{H}), 2.04(\mathrm{~s}, 3 \mathrm{H}), 1.75(\mathrm{q}, J=7.6 \mathrm{~Hz}, 2 \mathrm{H}), 1.15(\mathrm{t}, J=7.2 \mathrm{~Hz}, 3 \mathrm{H}), 1.09(\mathrm{t}, J=7.2 \mathrm{~Hz}, 3 \mathrm{H}) ;{ }^{13} \mathrm{C} \mathrm{NMR}$ $\left(400 \mathrm{MHz}, \mathrm{CDCl}_{3}\right) \delta 172.0,171.1,135.9,124.8,65.4,42.2,40.3,32.4,32.0,24.7,21.2,14.6,13.3 ;$ IR (film, $\mathrm{cm}^{-1}$ ): 2974, 2936, 2878, 1740, 1641, 1433, 1233; HRMS (ESI) $\mathrm{m} / z$ calc'd for $\mathrm{C}_{13} \mathrm{H}_{23} \mathrm{NO}_{3}[\mathrm{M}+\mathrm{H}]^{+}$: 242.1756, found 242.1747 .

Entry 10: 48h. run 1: [L:B] = >99:1, $[E: Z]=13: 1 ;$ run 2: [L:B] = >99:1, [E:Z] = 12:1; average: [L:B] = >99:1, $[E: Z]=13: 1$. Purification by flash chromatography (15\% EtOAc/hexanes eluent) provided the desired linear acetate as the major product (run 1: $114.1 \mathrm{mg}, 0.65 \mathrm{mmol}, 65 \%$; run 2: $112.1 \mathrm{mg}, 0.64 \mathrm{mmol}$, 64\%; average yield: $65 \%)$. After column: $[\mathrm{L}: \mathrm{B}]=>99: 1 ;[E: Z]=13: 1$.

${ }^{1} \mathrm{H}$ NMR $\left(400 \mathrm{MHz}, \mathrm{CDCl}_{3}\right) \delta 7.39(\mathrm{~m}, 2 \mathrm{H}), 7.32(\mathrm{~m}, 2 \mathrm{H}), 7.27(\mathrm{~m}, 1 \mathrm{H}), 6.65(\mathrm{~d}, J=15.6 \mathrm{~Hz}, 1 \mathrm{H})$, $6.29(\mathrm{dt}, J=15.8,6.4 \mathrm{~Hz}, 1 \mathrm{H}), 4.73(\mathrm{dd}, J=6.8,1.6 \mathrm{~Hz}, 2 \mathrm{H}), 2.1(\mathrm{~s}, 3 \mathrm{H}) ;{ }^{13} \mathrm{C} \mathrm{NMR}\left(100 \mathrm{MHz}^{\mathrm{C}} \mathrm{CDCl}\right) \delta$ $170.8,136.2,134.2,128.6,128.0,126.6,123.1,65.0,21.0 ;$ IR (film, $\left.\mathrm{cm}^{-1}\right)$ : 3082, 3059, 3028, 2943, 2882, 1738, 1495, 1449, 1379, 1364, 1233; HRMS (EI) $m / z$ calc'd for $\left[\mathrm{C}_{11} \mathrm{H}_{12} \mathrm{O}_{2}\right]^{+}$: 176.0837, found 176.0841 .

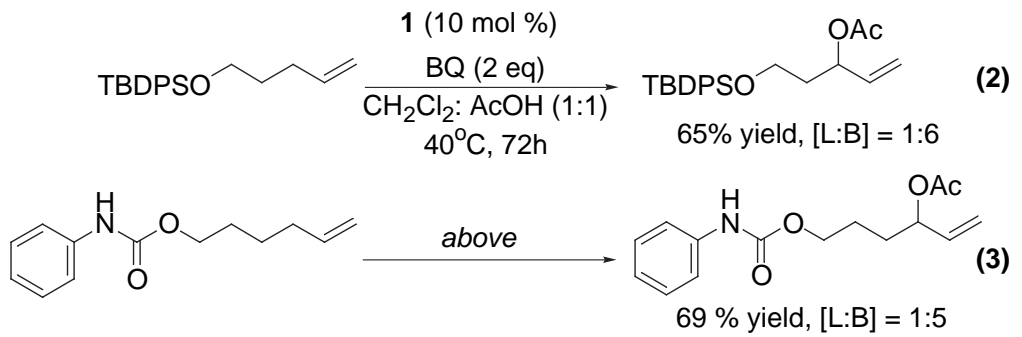

General procedure for catalyst 1 (equations 2, 3): A $40 \mathrm{~mL}$ borosilicate glass vial was charged with the following solids: catalyst 1 (53.2 $\mathrm{mg}, 0.1 \mathrm{mmol}, 10 \mathrm{~mol} \%)$, benzoquinone $(217 \mathrm{mg}, 2.0 \mathrm{mmol}, 2 \mathrm{eq})$. A separate 4 $\mathrm{mL}$ borosilicate glass vial was charged with olefin substrate $(0.1 \mathrm{mmol})$ which was diluted $\mathrm{with}^{\mathrm{C}} \mathrm{CH}_{2} \mathrm{Cl}_{2}$ and transferred to the solids vial via pipette $\left(3 \times 1 \mathrm{~mL}\right.$, total volume of $\mathrm{CH}_{2} \mathrm{Cl}_{2}$ was always $\left.3 \mathrm{~mL}\right)$. The $40 \mathrm{~mL}$ vial was charged with $\mathrm{AcOH}(3 \mathrm{~mL})$, a stir bar, capped and allowed to heat at $40^{\circ} \mathrm{C}$. Aliquots were taken $(\sim 50 \mu \mathrm{L}$ filtered through a silica plug with $\left.\mathrm{Et}_{2} \mathrm{O}\right)$ at $\mathrm{t}=24 \mathrm{~h}, 48 \mathrm{~h}$, and $72 \mathrm{~h}$ to determine linear:branched ratios [L:B]. The reaction was quenched with saturated $\mathrm{NH}_{4} \mathrm{Cl}(10 \mathrm{~mL})$ and extracted with $\mathrm{CH}_{2} \mathrm{Cl}_{2}(3 \mathrm{x} 100 \mathrm{~mL})$. The combined organic layers were washed with $\mathrm{H}_{2} \mathrm{O}(2 \times 100 \mathrm{~mL})$, dried over $\mathrm{MgSO}_{4}$, filtered and concentrated in vacuo to give a brown/red oil. Purification of the crude by flash chromatography afforded a mixture of the branched and linear $(E)$-allylic acetates (trace amounts of the $Z$ isomer was detected by ${ }^{1} \mathrm{H}$ NMR) with the branched allylic acetate as the major product.

Equation 2: $72 \mathrm{~h}$. run 1: [L:B] = 1:6, run 2: [L:B] = 1:5.5, average reported: [L:B] = 1:6 $(24 \mathrm{~h},[\mathrm{~L}: \mathrm{B}]=1: 7,48 \mathrm{~h}$, $[\mathrm{L}: \mathrm{B}]=1: 6)$. Purification by flash chromatography (3\% EtOAc/hexanes eluent) provided the branched allylic acetate as the major product (run 1: $256.5 \mathrm{mg}, 0.67 \mathrm{mmol}, 67 \%$; run 2: $240.8 \mathrm{mg}, 0.63 \mathrm{mmol}$, 63\%; average yield reported: $65 \%)$. After column $[\mathrm{L}: \mathrm{B}]=1: 6$.

${ }^{1} \mathrm{H}$ NMR $\left(400 \mathrm{MHz}, \mathrm{CDCl}_{3}\right) \delta 7.65(\mathrm{~m}, 4 \mathrm{H}), 7.40(\mathrm{~m}, 6 \mathrm{H}), 5.78(\mathrm{ddd}, J=17.0,10.4,6.0 \mathrm{~Hz}, 1 \mathrm{H})$, $5.46(\mathrm{~m}, 1 \mathrm{H}), 5.23(\operatorname{app~dt}, J=17.2,1.2 \mathrm{~Hz}, 1 \mathrm{H}), 5.15($ app dt, $J=10.0,1.6 \mathrm{~Hz}, 1 \mathrm{H}), 3.69(\mathrm{t}, J=6.4 \mathrm{~Hz}$, $2 \mathrm{H}), 2.0(\mathrm{~s}, 3 \mathrm{H}), 1.87(\mathrm{~m}, 2 \mathrm{H}), 1.05(\mathrm{~s}, 9 \mathrm{H}) ;{ }^{13} \mathrm{C} \mathrm{NMR}\left(100 \mathrm{MHz}, \mathrm{CDCl}_{3}\right) \delta 170.1,136.4,135.56$ and 135.53 (d), 133.68 and 133.6 (d), 129.6, 127.6, 116.5, 71.9, 59.7, 37.0, 26.8, 21.2, 19.1; IR (film, $\mathrm{cm}^{-1}$ ): 3073, 3050, 3000, 2957, 2932, 2891, 2859, 1742, 1474, 1427, 1371, 1236, 1111; HRMS (CI, NH $\mathrm{NH}_{3} \mathrm{z}$ calc'd for $\mathrm{C}_{23} \mathrm{H}_{34} \mathrm{NO}_{3} \mathrm{Si}\left[\mathrm{M}+\mathrm{NH}_{4}\right]^{+}:$400.2308, found 400.2308.

Equation 3: $72 \mathrm{~h}$. run 1,2: $[\mathrm{L}: \mathrm{B}]=1: 5(24 \mathrm{~h}, \quad[\mathrm{~L}: \mathrm{B}]=1: 7 ; \quad \mathrm{t}=48 \mathrm{~h}, \quad[\mathrm{~L}: \mathrm{B}]=1: 7)$. Purification by flash chromatography (12\% EtOAc/hexanes eluent) provided the branched allylic acetate as the major product (run 1: $190.4 \mathrm{mg}, 0.69 \mathrm{mmol}, 69 \%$; run 2: $189.9 \mathrm{mg}, 0.68 \mathrm{mmol}, 68 \%$; average yield reported: 69\%). After column $[\mathrm{L}: \mathrm{B}]=1: 5$.

${ }^{1} \mathrm{H}$ NMR $\left(400 \mathrm{MHz}, \mathrm{CDCl}_{3}\right) \mathrm{d} 7.38(\mathrm{brd}, J=7.6 \mathrm{~Hz}, 2 \mathrm{H}), 7.30(\mathrm{t}, J=7.6 \mathrm{~Hz}, 2 \mathrm{H}), 7.05(\mathrm{t}, J=7.2 \mathrm{~Hz}$, $1 \mathrm{H}), 6.71(\mathrm{brs}, 1 \mathrm{H}), 5.78(\mathrm{ddd}, J=17.4,10.4,6.4,1 \mathrm{H}), 5.32(\mathrm{~m}, 1 \mathrm{H}), 5.25(\operatorname{app~dt}, J=17.2,1.6,1 \mathrm{H}), 5.19$ (app dt, $J=10.4,1.2 \mathrm{~Hz}, 1 \mathrm{H}) 4.17(\mathrm{t}, J=6.0 \mathrm{~Hz}, 2 \mathrm{H}), 2.07(\mathrm{~s}, 3 \mathrm{H}), 1.73(\mathrm{~m}, 4 \mathrm{H}) ;{ }^{13} \mathrm{C} \mathrm{NMR}(100 \mathrm{MHz}$, $\left.\mathrm{CDCl}_{3}\right) \delta 170.5,153.4,137.8,136.1,129.0,123.4,118.6,117.0,74.2,64.8,30.8,24.6,21.2 ; \mathrm{IR}\left(\mathrm{film}, \mathrm{cm}^{-1}\right): 3329$ 
(br), 3196, 3138, 3063, 2957, 2859, 1734 (br, shoulder 1716), 1601, 1539, 1225; HRMS (EI) $m / z$ calc'd for $\left[\mathrm{C}_{15} \mathrm{H}_{19} \mathrm{NO}_{4}\right]^{+}:$277.1314, found 277.1318.

Synthesis of bis[chloro(1,2,3-trihapto-1-decene)palladium(II)]. ${ }^{11}$

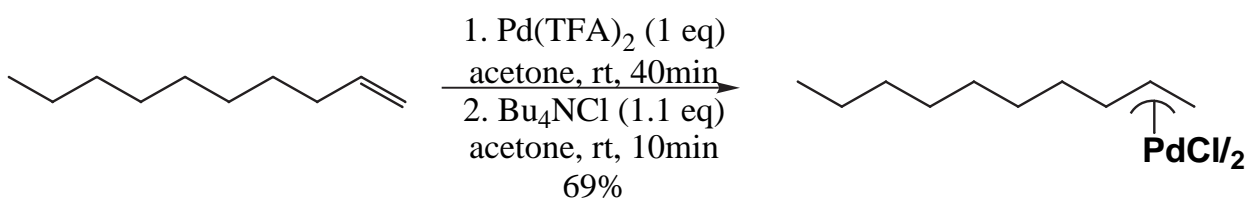

An oven dried $50 \mathrm{~mL}$ flask was charged with $\mathrm{Pd}(\mathrm{TFA})_{2}(472 \mathrm{mg}, 1.42 \mathrm{mmol})$ and a stir bar under $\mathrm{N}_{2}$. To this was added $12 \mathrm{~mL}$ of freshly distilled acetone (distilled over $\mathrm{K}_{2} \mathrm{CO}_{3}$ ) and 1-decene (200 mg, $270 \mu \mathrm{L}, 1.42 \mathrm{mmol}$ ). The reaction was allowed to stir for $40 \mathrm{~min}$ at $\mathrm{rt}$ under an atmosphere of Ar, at which time an acetone solution (3 $\mathrm{mL}$ ) of $n$ - $\mathrm{Bu}_{4} \mathrm{NCl}$ (434 mg, $1.56 \mathrm{mmol}$ ) was added via syringe. The anion exchange proceeded at $\mathrm{rt}$ for $15 \mathrm{~min}$. The mixture was filtered over a plug of Celite (to remove metallic Pd), concentrated and purified via column chromatography (50 $\%$ EtOAc/hexanes eluent) to afford bis[chloro(1,2,3-trihapto-1-decene)palladium (II)] as a yellow, oily solid (277mg, $0.98 \mathrm{mmol}, 69 \%)$.

${ }^{1} \mathrm{H}$ NMR (400 MHz, $\mathrm{CCl}_{4}$ doped with $\left.\mathrm{C}_{6} \mathrm{D}_{6}\right) \delta 4.92(\mathrm{td}, J=11.6,6.8 \mathrm{~Hz}, 1 \mathrm{H}, \mathrm{H}-2), 3.59(\mathrm{~d}, J=6.4 \mathrm{~Hz}, 1 \mathrm{H}$, anti H-1), 3.51 (m, 1H, H-3), 2.5 (d, $J=11.6,1 \mathrm{H}$, syn H-1), 1.6-1.1 (m, $12 \mathrm{H}), 0.79$ (t, $J=6.4 \mathrm{~Hz}, 3 \mathrm{H})$.
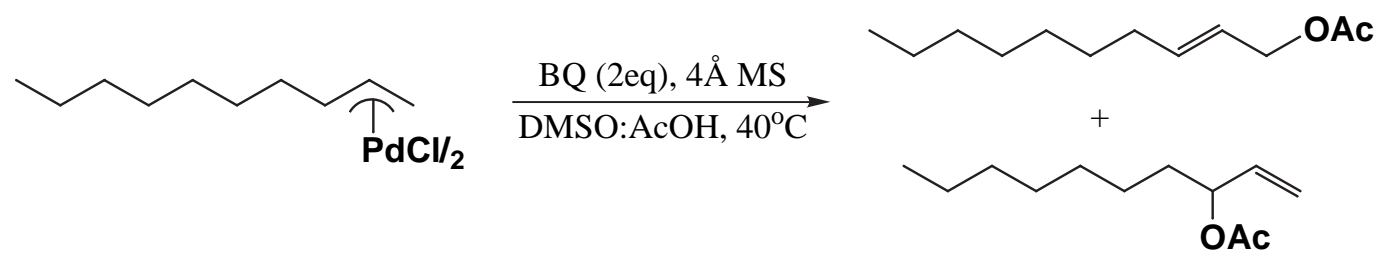

A $2 \mathrm{~mL}$ borosilicate vial was charged with $28 \mathrm{mg}(0.1 \mathrm{mmol})$ of bis[chloro(1,2,3-trihapto-1-decene)palladium (II)] and a magnetic stir bar. A separate $2 \mathrm{~mL}$ vial was charged with the following: BQ $(21.7 \mathrm{mg}, 0.2 \mathrm{mmol}), 4 \AA \mathrm{MS}(22$ $\mathrm{mg})$, DMSO $(0.3 \mathrm{~mL})$, and $\mathrm{AcOH}(0.3 \mathrm{~mL})$. The liquid mixture was transferred via pipette into the vial charged with bis[chloro(1,2,3-trihapto-1-decene)palladium (II)] and the solution was heated at $40^{\circ} \mathrm{C}$. Aliquots were take in $(\sim 10$ $\mu \mathrm{L}$ filtered with $\mathrm{Et}_{2} \mathrm{O}$ through a short pipette plug of silica) at $\mathrm{t}=1,3$ and $24 \mathrm{~h}$. Linear $(E)$-allylic acetate and branched allylic acetate were formed at $24 \mathrm{~h}$ in $24 \%$ and $18 \%$ yields, respectively, based on GC analysis using standard curves with authentic acetates and nitrobenzene.

\section{References:}

${ }^{1}$ Still, W.C.; Kahn, M.; Mitra, A. J. Org. Chem. 1978, 43, 2923.

${ }^{2}$ Lucas, R.C.; Liu, S.; Newlands, M.J.; Gabe, E. Can. J. Chem. 1990, 68, 1357.

${ }^{3}$ (a). Bell, E. V.; Bennett, G.M. J. Chem. Soc. 1928, 3189; (b). Gasparrini, F.; Giovannoli, M.; Misiti, D.; Natile, G.; Palmieri, G. Tetrahedron 1984, 40, 165.

${ }^{4} \mathrm{~S}-\mathrm{Pd}$ coordination mode is tentatively assigned based on other Pd(II) bis-sulfoxide complexes and Pd(II) DMSO complexes: (1) Bennett, M.J.; Cotton, F.A.; Weaver, D.L. Nature. 1966, 212, 286. (2) Pettinari, C.; Pellei, M.;

Cavicchio, G. Crucianelli, M.; Panzeri, W.; Colapietro, M.; Cassetta, A. Organometallics 1999, $18,555$.

${ }^{5}$ Dauben, W.G.; Wolf, R.E. J. Org. Chem. 1970, 35, 2361.

${ }^{6}$ Brust, D. P.; Tarbell, D. S. J. Org. Chem. 1966, 31, 1251.

${ }^{7}$ Nemoto, H.; Shiraki, M.; Fukumoto. J. Org. Chem. 1996, 61, 1347.

${ }^{8}$ Lowik, D.W.P.M.; Liskamp, R.M.J. Eur. J. Org. Chem. 2000, 1219.

${ }^{9}$ Breit, B.; Seiche, W. J. Am. Chem. Soc. 2003, 125, 6608.

${ }^{10}$ Musa, O.M.; Choi, S.-Y.; Horner, J.H.; Newcomb, M. J. Org. Chem. 1998, 63, 786.

${ }^{11}$ Trost, B.M.; Metzner, P.J. J. Am. Chem. Soc. 1980, 102, 3572. 